\title{
Brain glucose utilization in systemic lupus erythematosus with neuropsychiatric symptoms: a controlled positron emission tomography study
}

\author{
A. Otte 1,3, S.M. Weiner², H.H. Peter², J. Mueller-Brand ${ }^{1}$, M. Goetze1, E. Moser3, J. Gutfleisch², S. Hoegerle³, \\ F.D. Juengling ${ }^{3}$, E.U. Nitzsche ${ }^{3}$ \\ 1 Institute of Nuclear Medicine, University Hospital, Basel, Switzeriand \\ 2 Department of Rheumatology and Immunology, University Hospital Freiburg, Germany \\ ${ }^{3}$ Department of Nuclear Medicine, University Hospital Freiburg, Germany
}

Received 28 February and in revised form 2 April 1997

\begin{abstract}
In contrast to morphological imaging [such as magnetic resonance imaging (MRI) or computed tomography], functional imaging may be of advantage in the detection of brain abnormalities in cases of neuropsychiatric systemic lupus erythematosus (SLE). Therefore, we studied 13 patients (aged $40 \pm 14$ years, 11 female, 2 male) with neuropsychiatric SLE who met four of the American Rheumatism Association criteria for the classification of SLE. Ten clinically and neurologically healthy volunteers served as controls (aged $40 \pm 12$ years, 5 female, 5 male). Both groups were investigated using fluorine-18-labelled fluorodeoxyglucose brain positron emission tomography (PET) and cranial MRI. The normal controls and 11 of the 13 patients showed normal MRI scans. However, PET scan was abnormal in all 13 SLE patients. Significant group-to-group differences in the glucose metabolic index (GMI=region of interest uptake/global uptake at the level of the basal ganglia and thalamus) were found in the parieto-occipital region on both sides: the GMI of the parieto-occipital region on the right side was $0.922 \pm 0.045$ in patients and $1.066 \pm 0.081$ in controls $(P<0.0001$, Mann Whitney $U$ test), while on the left side it was $0.892 \pm 0.060$ in patients and $1.034 \pm 0.051$ in controls $(P=0.0002)$. Parietooccipital hypometabolism is a conspicuous finding in mainly MRI-negative neuropsychiatric SLE. As the parieto-occipital region is located at the boundary of blood supply of all three major arteries, it could be the most vulnerable zone of the cerebrum and may be affected at an early stage of the cerebrovascular disease.
\end{abstract}

Key words: Neuropsychiatric systemic lupus erythematosus - Positron emission tomography - Fluorine-18 fluorodeoxyglucose - Parieto-occipital brain hypometabolism

Correspondence to: A. Otte, Institute of Nuclear Medicine, Department of Radiological Science, University Hospital, School of Medicine, Petersgraben 4, CH-4031 Basel, Switzerland
Eur J Nucl Med (1997) 24:787-791

\section{Introduction}

Studies with positron emission tomography (PET) and single-photon emission tomography (SPET) have shown that in certain brain disorders functional imaging is more sensitive than morphological imaging such as magnetic resonance imaging (MRI) and X-ray computed tomography. This applies to traumatic brain injury [1-8], whiplash injury $[9,10]$, migraine [11], or Alzheimer's disease $[12,13]$, and also neuropsychiatric systemic lupus erythematosus (SLE) [14-16]. Neuropsychiatric SLE has in most cases been studied in late stages of the disease; little is known of early neuropsychiatric SLE. There are conflicting reports regarding the regions involved in neuropsychiatric SLE: using azetazolamide-enhanced SPET in an SLE patient with preclinical central nervous system (CNS) involvement, Grünwald et al. [14] showed a marked reduction in the cortical perfusion reserve, particularly in both frontal lobes, whereas Kovacs et al. [17] showed various other abnormal regions such as the occipital cortex or the basal ganglia in cases of active neuropsychiatric SLE. Szer et al. [18] reported on a girl with a concurrent ischaemic event involving both parietal lobes. Therefore, we wondered whether there is a typical pattern of metabolism in neuropsychiatric SLE, reflecting the vulnerability of the different zones in the cerebrum.

\section{Materials and methods}

Each study participant signed an informed consent form, explaining the investigative nature of the study, its risks, and its merits.

Study population. Ten normal controls (mean age $40 \pm 12$ years, 5 female, 5 male) and 13 patients (mean age $40 \pm 14$ years, 11 fe- 
male, 2 male) were studied. All patients met four of the American Rheumatism Association criteria for the classification of SLE [19]. All study participants were interviewed with regard to headache, vertigo, visual symptoms (blurred vision, scintillating scotoma, oscillopsia), impaired memory and/or concentration, depression, sleep disturbances, anxiety and instability [20].

None of the study participants exhibited anamnestic hints of neurovascular or neurodegenerative diseases. Secondary causes such as infection, uraemia, hypertension, drugs and metabolic abnormalities were excluded. Eleven of the 13 neuropsychiatric SLE patients received corticosteroid treatment and three received immunosuppression with cyclophosphamide or azathioprine. As regards the control group, only participants with normal clinical and neurological findings were included in the study. For the patient group, only those patients were included who showed clinical and neurological findings indicating CNS involvement by the disease. Neuropsychiatric manifestation due to SLE was defined as an abnormality of neurological function identified by current history and physical examination and noted as a change from a prior state, if this abnormality could not be attributed to any cause other than the disease process itself. The CNS manifestations were classified into "major" (seizures, focal motor or sensory deficits, generalized disturbances, psychosis, organic brain syndrome) and "minor" signs (paraesthesia or clumsiness without objective findings, headache, cognitive disorders) according to the classification of How et al. [21]. Nine patients with "initial" CNS SLE had shown neuropsychiatric signs for no longer than 1 year, whereas four patients with "manifest" CNS disease had been symptomatic for more than 1 year. All patients diagnosed as having manifest CNS SLE ( $n=4)$ had one or more major signs, and two of them showed pathological findings on MRI. Patients in the initial CNS SLE group had one major sign $(n=5)$ or one to three minor signs $(n=4)$.

MRI of the brain was also undertaken in all patients and controls.

The following neuropsychiatric symptoms were observed in the SLE patients:

- Impairment of memory and of intelligent function: $n=6$ patients

- Psychosis: $n=4$

- Seizure: $n=1$

- Focal motor deficits: $n=2$

- Persistent headache: $n=4$

- Paraesthesia: $n=2$

- Vertigo: $n=3$

- Impairment of orientation and perception: $n=1$

- Visual symptoms: $n=4$

- Recurrent apoplexy: $n=1$

The following concomitant clinical and serological parameters were observed in the SLE patients:

- Positive antinuclear antibodies: $n=13$ patients

- Positive anti-ds DNA: $n=7$

- Positive antibodies against extractable nuclear antigen: $n=10$

- Phospholipid antibodies: $n=6$

- Cutaneous lesions: $n=7$

- Lupus nephritis: $n=8$

- Arthralgia: $n=6$

- Myalgia: $n=4$

- Sicca complex: $n=4$

- Cytopenia: $n=4$

Radiopharmaceuticals. All participants were injected with $370 \mathrm{MBq}$ fluorine-18 fluorodeoxyglucose (FDG) into a peripheral vein. FDG was synthesized and prepared as reported previously [22].

PET study protocol. Before the start of the PET procedure, each study participant had to fast for at least $12 \mathrm{~h}$ before the study and to empty the bladder. Then, during the 10 -min waiting period prior to injection, patients and controls were placed on a bed in the preparation area in a quiet room with dim lights, remaining awake, but with closed eyes. After injection, patients and controls remained on the bed for another $30 \mathrm{~min}$. The head of each subject was then positioned parallel to the orbitomeatal line with the aid of a laser beam, after which it was placed in a moulded thermoplastic head support to minimize head movements during the scanning procedure. All subjects were studied at rest. The acquisition started about 35 min p.i. PET imaging was performed on a Siemens CTI ECAT EXACT tomograph. This device records 31 planes simultaneously, which encompass a $10.8-\mathrm{cm}$ field of view. The spatial resolution is $6.0 \mathrm{~mm}$ full-width at half-maximum. Photon attenuation was automatically calculated using SiemensCTI standard software [23]. A total of six frames, each of $5 \mathrm{~min}$ duration, were acquired. Images were reconstructed using filtered back-projection (Shepp-Logan filter, cut-off 0.3 cycles/pixel). For data analysis, summed images were used (summing the dynamically acquired frames $1-6$ into one image).

Data analysis. Standardized cortical and subcortical elliptical regions of interest (ROIs) were assigned to three adjacent transaxial slices of the following regions by consensus of two independent investigators:

1. Frontal

2. Parietal

3. Occipito-frontal

4. Temporo-lateral

5. Temporo-mesial

6. Parieto-occipital (P-O)

7. Thalamus

8. Putamen

9. Caudate nucleus

10. Cerebellar (mean of lateral and dorsal cerebellum)

11. Brain stem

12. Global at the level of the thalamus and basal ganglia

The shape and size of ROIs were the same in all patients and controls, since the ROI templates were copied onto the corresponding planes of each study. The shape and size of global ROIs were fitted to actual head form by manipulation. The position of all ROIs was adjusted to the corresponding anatomical localization. In each ROI the average uptake per pixel per time was determined.

ROI analysis was performed using a SUN sparc station and SUNVIEW software. To allow inter-individual comparisons, regional cerebral metabolism was normalized by global glucose metabolism (glucose metabolic index, GMI; GMI=ROI uptake/global uptake) determined from the global brain ROI at the level of the thalamus and basal ganglia.

Statistical analysis. All data are expressed as mean \pm 1 SD. Groupto-group differences were tested using the Mann Whitney $U$ test (M.W.) and the software StatView 4.10. 


\section{Results}

MRI of the brain showed no pathological findings in any of the controls or in 11 of the 13 patients. The two patients with pathological MRI findings had had organic neuropsychiatric symptoms for more than 1 year. In con-
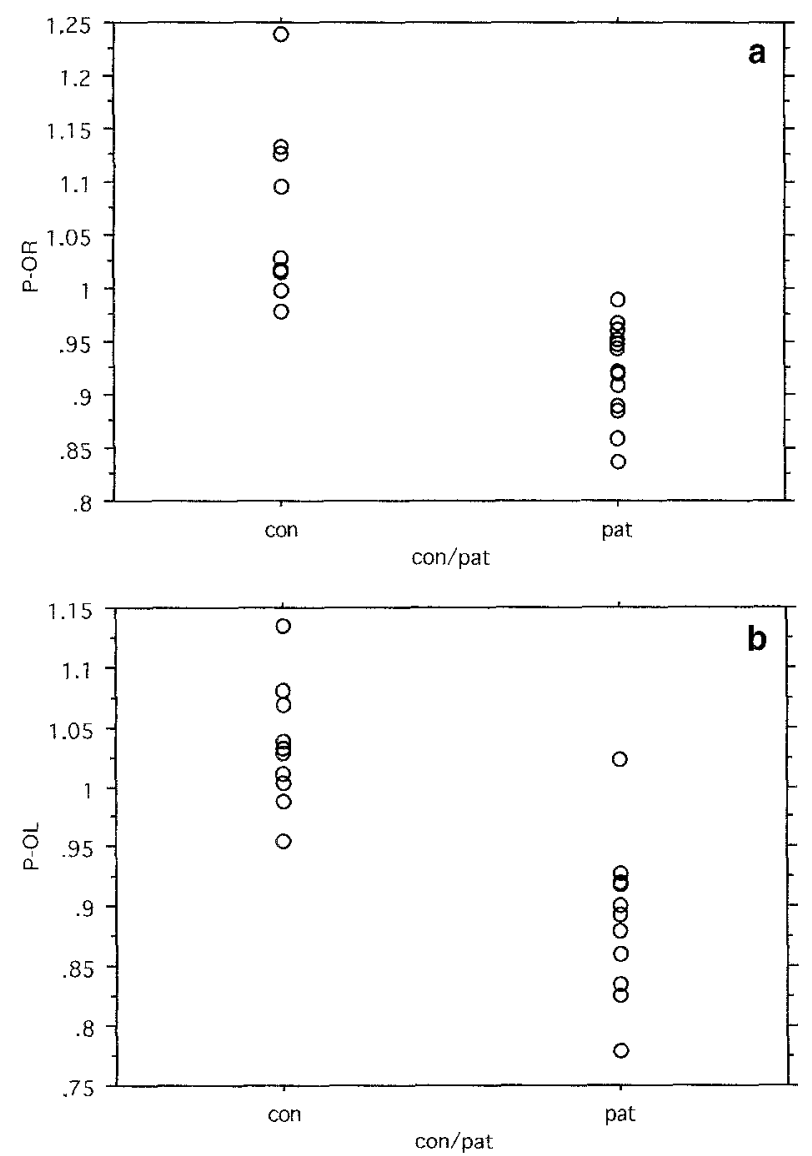

Fig. 1. Scattergrams of GMI values of P-O on the right (a) and left (b) sides, in the control group (con, $n=10$ ) and the patient group (pat, $n=13$ ) trast, the patient group revealed a significant hypometabolism in the P-O regions [on the right (R) and left (L) side] as compared to the control group (Fig. 1):

- GMI P-O R: controls $1.066 \pm 0.081$, patients $0.922 \pm 0.045 ; P<0.0001$

- GMI P-O L: controls $1.034 \pm 0.051$, patients $0.892 \pm 0.060 ; P=0.0002$

Group-to-group differences in regions other than the P-O were not statistically significant. However, careful evaluation of the individual metabolic pattern did additionally reveal, in six of the 13 patients (46\%), GMI values which were more than one or two standard deviations below normal control values in regions other than the P-O. In one patient (no. 5), P-O hypometabolism was combined with a patchy uptake pattern over the whole brain, while in another (no. 1) it was combined with a relative hypermetabolism in the basal ganglia and thalami (Table 1).

Furthermore, when the SLE patient group was divided into patients who showed CNS involvement for the first time at admission (group 1, $n=9$ ) and patients with organic neuropsychiatric symptoms for more than 1 year (group 2,n=4), no significant difference was found between groups 1 and 2 with regard to glucose utilization in any of the analysed brain regions.

\section{Discussion}

Our study indicates that neuropsychiatric SLE - irrespective of the stage of CNS involvement (i.e. initial or non-initial) - has a typical pattern of hypometabolism in the $\mathrm{P}-\mathrm{O}$ region, although non-P-O regions may also be affected. Since the P-O region is located at the boundary of supply of all three major arteries, it is thought to be the most vulnerable zone of the cerebrum [24]. There-

Table 1. Brain PET findings in patients

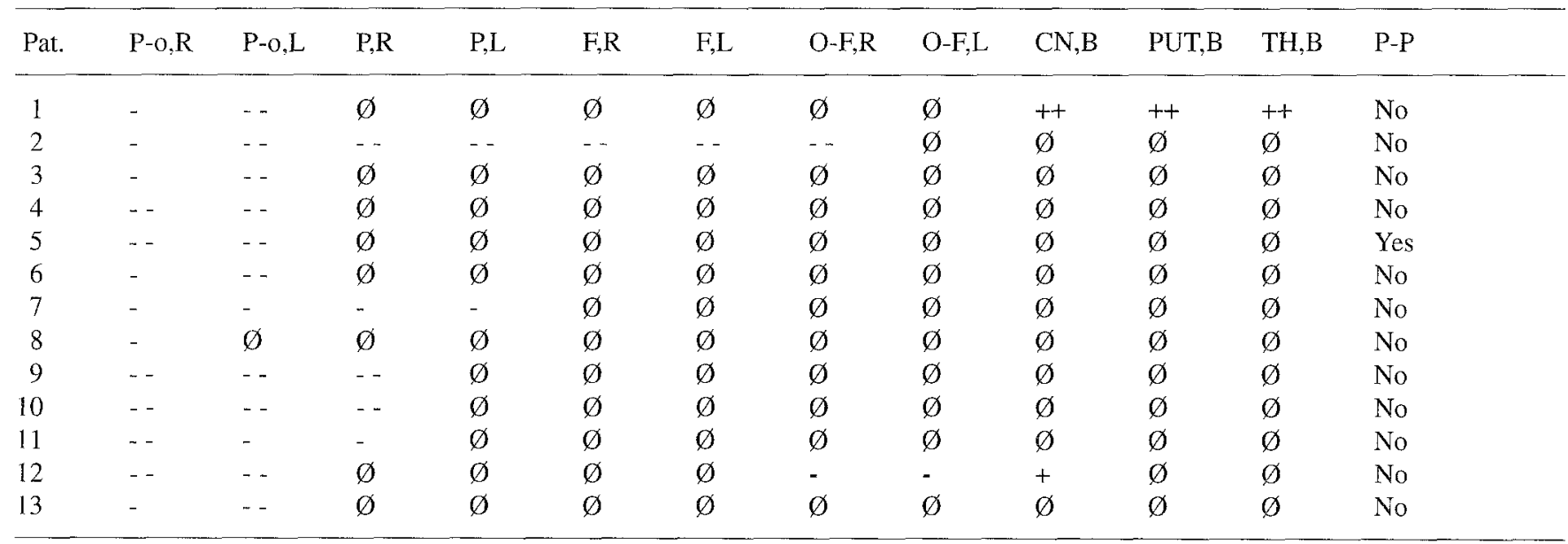

P-O, Parieto-occipital; P, parietal; F, frontal; O-F, occipito-frontal; CN, caudate nucleus; PUT, putamen; TH, thalamus; P-P, patchy uptake pattern; R, right brain side; $\mathrm{L}$, left brain side; $\mathrm{B}$, both sides. -, GMI $>1$ SD below normal controls; - -, $>2$ SD; +, GMI $>1$ SD above normal controls;,$++>2 \mathrm{SD} ; \varnothing$, GMI within normal limits. 
fore, we think that this region is frequently affected at an early stage of SLE with suspected CNS involvement. MRI findings are inconspicuous in early neuropsychiatric SLE, in contrast to the well-known MRI findings in manifest and chronic CNS SLE [25-27]; against this background the information provided by our PET data might have important diagnostic and therapeutic implications at an early stage of the disease. However, the question of whether vasculitis or infarctions and haemorrhages in this $\mathrm{P}-\mathrm{O}$ zone are the consequence or a cause of the SLE process remains open.

Abnormalities in the P-O region are not pathognomonic for neuropsychiatric SLE: they can also be found, for example, in the late whiplash syndrome $[9,10,28]$, in dementia of the Alzheimer type [12], in the elderly [13], in sleep apnea syndrome [13] and following cerebral hypoxia [13]. However, after careful clinical and serological classification of our neuropsychiatric SLE patients, these differential diagnoses could be excluded.

In studies on the pathology of cerebral lupus, vasculopathy, infarctions and haemorrhages are often observed, whereas vasculitis is rare [29]. Furthermore, the concept of hypercoagulability in SLE patients has diverted the direction of therapy from immunosuppression [30] (as in the majority of our patients) towards anticoagulation [31]. Randomized trials have commenced in order to determine the optimal mode of therapy for SLE patients under strict inclusion criteria, based on well-defined patient categories. Functional imaging like PET, or SPET, could be a powerful tool for such an enterprise.

\section{References}

1. Bavetta S, Nimmon CC, White J, McCabe J, Huneidi AH, Bomanji J, Birkenfeld B, Charlesworth M, Britton KE, Greenwood RJ. A prospective study comparing SPET with MRI and $\mathrm{CT}$ as prognostic indicators following severe closed head injury. Nucl Med Commun 1994; 15: 961-968.

2. Masdeu JC, VanHerretum RL, Kleiman A, Anselmi G, Kissane K, Horng J, Yudd A, Luck D, Grundman M. Early single photon emission computed tomography in mild head trauma, a controlled study. J Neuroimag 1994; 4: 177--181.

3. Prayer L, Wimberger D, Oder W, Kramer J, Schindler E, Podreka I, Imbof H. Cranial MR imaging and cerebral $99 \mathrm{mTc}$ HMPAO SPECT in patients with subacute or chronic severe closed head injury and normal CT examinations. Acta Radiol 1993; 34: 593-599.

4. Newton MR, Greenwood RJ, Britton KE. A study comparing SPECT with CT and MRI after closed head injury. $J$ Neurol Neurosurg Psychiatry 1992; 55: 92-94.

5. Roper SN, Mena I, King WA, Schweitzer J, Garrett K, Mehninger CM, McBride D. An analysis of cerebral blood flow in acute closed head injury using technetium-99m-HMPAO SPECT and computed tomography. J Nucl Med 1991; 32 : 1684-1687 (erratum 2070).

6. Nedd K, Sfakianakis G, Ganz W, Uricchio B, Vernberg D, Villanueva P, Jabir AM, Bartlett J, Keena J. 99mTc-HMPAO SPECT of the brain in mild to moderate traumatic brain injury patients compared with CT - a prospective study. Brain Inj 1993; 7: 469-479.
7. Gray BG, Ichise M, Chung DG, Kirsh JC, Franks W. Technetium-99m-HMPAO SPECT in the evaluation of patients with a remote history of traumatic brain injury: a comparison with $\mathrm{x}$ ray computed tomography. $J$ Nucl Med 1992; 33: 52-58.

8. Yamakami I, Yamaura A, Isobe K. Types of traumatic brain injury and regional cerebral blood flow assessed by $99 \mathrm{~m}$ TcHMPAO SPECT. Neurol Med Chir 1993; 33: 7-12.

9. Otte A, Mueller-Brand J, Fierz L. Brain SPECT findings in late whiplash syndrome. Lancet 1995; 345: 1513-1514.

10. Otte A, Ettlin TM, Fierz L, Mueller-Brand J. Parieto-occipital hypoperfusion in late whiplash syndrome: first quantitative SPECT study using Tc-99m-bicisate (ECD). Eur J Nucl Med 1996; 23: 72-74.

11. Friberg L. Cerebral blood flow changes in migraine: methods, observations and hypotheses. J Neurol 1991; 238 Suppl 1: S12-S17.

12. Waldemar G, Bruhn P, Kristensen M, Johnsen A, Paulson O, Lassen NA. Heterogeneity of neocortical cerebral blood flow deficits in dementia of the Alzheimer type: a 99mTc-HMPAO-SPECT study J Neurol Neurosurg Psychiatry 1994; 57: 285--295.

13. Miller BL, Mena I, Daly J, Gombetti RJ, Goldberg MA, Lesser I, Garetti K, Villanueva-Meyer J, Liu CK. Temporo-parietal hypoperfusion with single photon emission computerized tomography in conditions other than Alzheimer's disease. Dementia 1990; 1: 41-45.

14. Grünwald F, Schomburg A, Badali A, Ruhlmann J, Pavics L, Biersack HJ. ${ }^{18}$ FDG PET and azetaolamide-enhanced ${ }^{99} \mathrm{mTc}-$ HMPAO SPET in systemic lupus erythematosus. Eur $J$ Nucl Med 1995; 22: 1073-1077.

15. Sibbitt WL, Sibbitt RR. Magnetic resonance spectroscopy and positron emission tomography scanning in neuropsychiatric systemic lupus erythematosus. Rheum Dis Clin North Am 1993; 19: 851-868.

16. Stoppe G, Wildhagen K, Meyer GJ, Schober O. Use of fluorodeoxyglucose PET in the diagnosis of central nervous system lupus erythematosus and a comparison with CT and MRI. $\mathrm{Nu}$ klearmedizin 1989; 28: 187-192.

17. Kovacs JA, Urowitz MB, Gladman DD, Zeman R. The use of single photon emission computerized tomography in neuropsychiatric SLE: a pilot study. I Rheumatol 1995; 22: 1247-1253.

18. Szer IS, Miller JH, Rawlings D, Shaham B, Bernstein B. Cerebral perfusion abnormalities in children with central nervous system manifestations of lupus by single photon emission computed tomography. $J$ Rheumatol 1993; 20: 2143-2148.

19. Tan EM, Cohen AS, Fried JF, et al. The 1982 revised criteria for the classification of systemic lupus erythematosus (SLE). Arthritis Rheum 1982; 25: 1271-1277.

20. Ettlin T, Kischka U, Reichmann S, Radü EW, Heim S, a Wengen D, Benson FD. Cerebral symptoms after whiplash injury of the neck: a prospective clinical and neuropsychological study of whiplash injury. $J$ Neurol Neurosurg Psychiatry 1992; 55: $943-948$.

21. How A, Dent PB, Liao SK, Denburg JA. Antineuronal antibodies in neuropsychiatric systemic lupus erythematosus. Arthritis Rheum 1985; 28: 789-795.

22. Hamacher K, Coenen HH, Stöcklin G. Efficient stereospecific synthesis of no-carrier added $2-\left[{ }^{18} \mathrm{~F}\right]$-fluoro-2-deoxy-D-glucose using aminopolyether supported nucleophilic substitution. I Nucl Med 1986; 27: 235-238.

23. Huang SC, Carson R, Phelps M, Hoffman E, Schelbert H, Kuhl D. A boundary method for attenuation correction in positron emission tomography. IEEE Trans Nucl Sci 1981; 22: $627-637$. 
24. Graham DG, Brierly JB. Vascular disorders of the central nervous system. In: Adams J, ed. Neuropathology. London: Edward Arnold; 1984: 125-207.

25. Isshi K, Hirohata S, Hashimoto T, Miyashita H. Systemic lupus erythematosus presenting with diffuse low density lesions in the cerebral white matter on computed axial tomography scans: its implication in the pathogenesis of diffuse central nervous system lupus. $J$ Rheumatol 1994; 21: 1758-1762.

26. Gonzalez-Crespo MR, Blanco FJ, Ramos A, Ciruelo E, Mateo I, Lopez-Pino MA, Gomez-Reino JJ. Magnetic resonance imaging of the brain in systemic lupus erythematosus. $\mathrm{Br} \mathrm{J}$ Rheumatol 1995; 34: 1055-1060.

27. Baum KA, Hopf U, Nehrig C, Stover M, Schorner W. Systemic lupus erythematosus: neuropsychiatric signs and symptoms related to cerebral MRI findings. Clin Neurol Neurosurg 1993; 95: 29-34.
28. Otte A, Ettlin TM, Fierz L, Kischka U, Muerner J, MuellerBrand J. Brain perfusioin patterns in 136 patients with chronic symptoms after distorsion of the cervical spine using single photon emission computed tomography, technetium-99mHMPAO and technetium-99m-ECD: a controlled study. $J$ Vasc Invest $1997 ; 3: 1-5$.

29. vanDam AP. Diagnosis of pathogenesis of CNS lupus. Rheumatol Int 1991; 11: 1-11.

30. McInness PM, Schuttinga J, Sanslone WR, Stark SP, Klippel JH. The economic impact of treatment of severe lupus nephritis with prednisolone and cyclophosphamide. Arthritis Rheum 1994; 37: 1000-1006.

31. Bruyn GA. Controversies in lupus: nervous system involvement. Ann Rheun Dis 1995; 54: 159-167. 